\title{
Observability Matrix Condition Number in Design of Measurement Strategies
}

\author{
J A Wilson $^{\mathrm{a}^{*}}$ and S Y Guhe ${ }^{\mathrm{a}}$ \\ ${ }^{\text {a } U n i v e r s i t y ~ o f ~ N o t t i n g h a m ~}$ \\ School of Chemical, Environmental \& Mining Engineering \\ University Park, Nottingham, NG7 2RD, UK
}

\begin{abstract}
In the work presented here we aim to demonstrate the utility of the observability matrix condition number as a metric in developing optimum measurement strategies in a range of process engineering contexts via some new examples. To demonstrate use of the approach itself we consider controller tuning in the field to show how response parameters can be estimated with a minimum of carefully timed empirical measurements. In front-end process development also we show how timing of the minimal number of measurements needed for chemical kinetic parameter estimation can be decided, with potential savings in empirical laboratory resource overheads. In the context of equipment specification, we show how capital investment can be balanced against the quality of data delivered for plant operation with an example in selecting the number and location of temperature sensors in a batch distillation. Finally, constructing the observability matrix itself can be problematic, especially in non-linear applications where we propose, as a feasible approach, its direct calculation from simulated measurement responses and their sensitivity to the unknown state and parameters.
\end{abstract}

Keywords: Optimum sensor location, batch distillation, reaction kinetics estimation.

\section{Introduction}

In the work presented here we aim to demonstrate the utility of the concept of system observability (well established in the field of process dynamic systems identification and estimation) in situations involving decisions on measurement strategies which arise in the wider process engineering contexts of process development, equipment specification and plant operation. Optimisation in the design of chemical engineering plant measurement systems has been addressed recently, and perhaps most directly, by Muske and Georgakis (2003) who express the problem as a 'Pareto' optimisation balancing cost of sensors against process information obtained. They provide a reaction system example but a more detailed analysis and discussion on tubular reactors is given by Waldraff et al. (1998) who consider optimal placement of a single sensor, their stated motivation being to have the lowest possible number of sensors. By contrast, in the context of plant operation, and in particular in the separate area of batch distillation, we

* Author to whom correspondence should be addressed: j.a.wilson@ nottingham.ac.uk 
look at choice of the number and physical location of sensors in a multi-sensor system and we provide a basis to balance sensor capital investment against the quality of process information obtained. At a different level we also consider optimisation of effort in performing parameter estimations both in the process development and plant operating situations. The objective here is to obtain reliable estimates of unkown parameters based on a minimum of empirical measurements, since each measurement taken and every experiment conducted carry a high financial overhead in engineer and technician people-hours, chemical analysis, laboratory resources or interruption to plant operations and production. The examples we provide relate to reaction kinetics capture during process development and to controller tuning on operating plant.

\section{Methods}

The concept of system observability is well founded in the context of dynamic systems identification and estimation. In the linear, time invariant case $\mathbf{z}$, the system state of order $m$ (in general a set of true dynamic states augmented with unknown parameters) relates to $\mathbf{y}$, the available measurements, of order $n$, for example in discrete time, by

$\mathbf{z}(k+1)=\mathbf{A z}(k)+\mathbf{B u}(k)$

$\mathbf{y}(k+1)=\mathbf{H z}(k+1)$

The observability matrix $\mathbf{V}$ then follows (see for example Ray, 1981) as

$$
\mathbf{V}=\left(\mathbf{H}^{\prime}\left|\mathbf{A}^{\prime} \mathbf{H}^{\prime}\right| \mathbf{A}^{\prime 2} \mathbf{H}^{\prime}|\cdots| \mathbf{A}^{\prime N-1} \mathbf{H}^{\prime}\right)^{\prime}
$$

In essence $\mathbf{V}$ connects $\mathbf{z}(0)$, the initial unknown state, to the concatenated sequence of measurements acquired over $N$ samples, according to

$$
\left(\mathbf{y}^{\prime}(0)\left|\mathbf{y}^{\prime}(1)\right| \mathbf{y}^{\prime}(2)|\cdots| \mathbf{y}^{\prime}(N-1)\right)^{\prime}=\mathbf{V z}(0)
$$

The standard confirmatory test for observability is that the rank of $\mathbf{V}$ must equate to the order of $\mathbf{z}$ (i.e. $\mathbf{z}(0)$ must be deterministically calculable from some subset of $m$ measured values selected from the full $N \times n$ set captured). $\mathbf{V}$ can be found numerically or better, if the underlying system equations are known, analytically, which gives better insight into the influences of system parameters and behaviour on its observability.

In the case of non-linear systems, calculation of the transition matrices embedded in each partition ( $\mathbf{A}^{\mathrm{j}}$ in Equation 3) linking $\mathbf{z}(0)$ to the later state $\mathbf{z}(j)$, and thence to measurement sample $\mathbf{y}(j)$, must be calculated individually. In weakly non-linear cases the transition matrix over samples 0 to $j$ can be approximated as the product $\mathbf{A}(1) \ldots \mathbf{A}(j)$ where $\mathbf{A}(j)$ is locally linearised at sample j. For more non-linear cases (see Section 4) this approximation can fail. To address this here we propose that $\mathbf{V}$ be calculated directly as the sensitivity of each measurement in $\mathbf{y}(j)$ to the initial state and parameters in $\mathbf{z}(0)$. Thus column $i$ in the $j^{\text {th }}$ partition of $\mathbf{V}$ arises as $\mathbf{v}_{i}(j)$ where

$$
\mathbf{v}_{i}(j)=\frac{\partial \mathbf{y}(j)}{\partial z_{i}} \approx\left(\mathbf{y}_{i}(j)-\mathbf{y}_{0}(j)\right) / \delta z_{i}
$$

$\mathbf{y}_{i}(j)$ is the measurement set at sample $j$ if the state $z_{i}(0)$ is perturbed by $\delta z_{i} . \quad \mathbf{y}_{0}(j)$ is the measurement set at sample $j$ with $\mathbf{z}(0)$ unperturbed. Thus with $\mathbf{z}$ of order $m$ we require 
to run $m+1$ non-linear simulations. Numerical integrity of the resulting $\mathbf{V}$ relies on that of the underlying numerical integrations and the choice of $\delta z_{i}$.

For optimisation we also need a scalar metric of observability. After reviewing options, Waldraff et al. (1998) themselves use the $\mathbf{V}$ matrix minimum singular value, its grammian singular value and the Popov-Belevich-Hautus rank. Here we choose, as a simple, direct metric of numerical good-conditioning in Equation 5, the condition number of $\mathbf{V}$ (i.e. $\operatorname{cond}(\mathbf{V})$ ). The basic premise of our approach is that the most well posed calculation of $\mathbf{z}(0)$ by choice of a minimal subset of $m$ measurements arises with the minimum $\operatorname{cond}\left(\mathbf{V}_{\mathrm{m}}\right)$ where $\mathbf{V}_{\mathrm{m}}$ is square, being formed as the corresponding $m$ rows in $\mathbf{V}$. The following sections demonstrate application of this metric in three cases.

\section{An illustrative example: field tuning a controller}

Consider a parameter estimation problem to demonstrate the proposed approach controller tuning in the field. This requires use of performance criteria which can be quickly and easily measured on a recorded system response. The classic 4:1 decay ratio target is important as are response time criteria (settling or rise time - more complex criteria, e.g. integral error criteria, are too cumbersome for field use) but, with integral action present, too slow an integral action time can lead to slow underlying drift (see Fig. 1). This makes precise determination of decay ratio difficult, especially from a partial response (decay ratio found simply from peak values is inaccurate). Modelled as a first order/second order oscillatory element cascade initially at rest, the response in Fig. 1 takes the form

$$
\theta(t)=a_{1} e^{-a_{2} t} \sin \left(a_{3} t+\phi\right)+\beta e^{-a_{4} t}+a_{5}
$$

where the initial conditions require

$\theta(0)=0=a_{1} \sin (\phi)+\beta+a_{5}$

$\dot{\theta}(t)=0=-a_{1} a_{2} \sin (\phi)-a_{1} a_{3} \cos (\phi)-\beta a_{4}$

where unknown parameters $a_{1}, \ldots, a_{5}$ are to be found from the recorder chart. We need a minimum of five points (plus the initial value) for quick manual determination (statistical fitting to a larger data set is ruled out in the field). At which 5 instants on the chart should the response be sampled to deliver the most precise parameter values? A non-subjective choice is based on minimum $\operatorname{cond}\left(\mathbf{V}_{5}\right)$. By differentiating Equation 7 we can evaluate the differential in Equation 6 and hence the observability matrix $\mathbf{V}$. Each row corresponds to one sampled measurement. Minimising $\operatorname{cond}\left(\mathbf{V}_{5}\right)$ by choice of 5 rows from $\mathbf{V}$ yields the optimal measurement strategy shown as circles in Fig. 1. Intuitively one might choose peak values but to capture the frequency, damping and drift, the results suggest that intermediate sampling is more efficient.

\section{Optimum sampling strategy in process development data capture}

Consider now a case of optimising expenditure in front-end process development, specifically the capture of reaction kinetic data (i.e. parameter estimation) for use in 


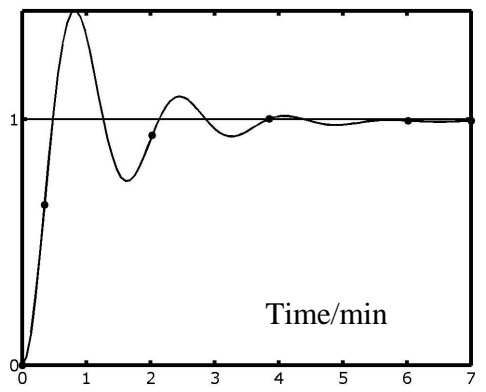

Figure 1: Optimum measurements for estimating a 5 parameter response.

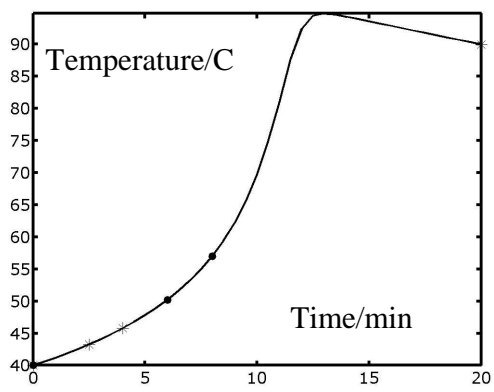

Figure 2:Optimum measurements for 2(•) and $3(*)$ kinetic parameter estimations.

laboratory or pilot plant studies or in plant equipment design. The example is motivated by experience in the 'high added value' batch process sector where development timescales are short and project resources are scarce.

We must determine a reaction rate constant and its temperature dependency (i.e. its Arrhenius parameters) with minimal laboratory bench-scale experimentation. Dewar Calorimetry provides a cheap and particularly rich source of reaction data (e.g. Mossebach and Reichert, 1997) where temperature response of an exothermic batch reaction is recorded in a near adiabatic, laboratory reactor. Typically, from an initial equilibrium, reaction is initiated by sudden release of one reagent (by smashing a vial inside the reactor). The initial reagent concentration $C(0)$ is known from the initial charge, i.e. in effect measured. The transient temperature $T(t)$ is recorded, typically taking the form in Figure 2.

We assume that the reaction order $\left(1^{\text {st }}\right)$, its enthalpy $H$ and other parameters (e.g. reactor and material heat capacities, heat transfer coefficient to the surroundings, temperature probe lags etc.) are all known a priori. The aim is to determine the Arrhenius coefficients $A, B$ defining the rate constant. Only a single material balance on the reacting component and a heat balance are needed. $\mathbf{z}$ is $4^{\text {th }}$ order $(T, C, A, B)$ ' so we can use the initial measurements, $T(0)$ and $C(0)$, plus a minimum of two other measurements of $T(t)$ to estimate the unknown parameters. To decide at what times these two measurements should be taken we can again construct the $\mathbf{V}$ matrix across the whole transient period and then select out measurement pairs to minimise $\operatorname{cond}\left(\mathbf{V}_{4}\right)$. In this case, the non-isothermal reaction is very highly non-linear in both state and parameters and so Equation 6 was used to calculate $\mathbf{V}$. Placement of the two transient temperature measurements that minimise $\operatorname{cond}\left(\mathbf{V}_{4}\right)$ is shown as circles in Fig. 2. Recall that the reaction enthalpy $H$ is known and use of measurements so early in the transient may arise from the need to 'see' the temperature rate dependency allowing discrimination between $A$ and $B$ while at the same time working where the reaction rate is high but not so high as to cause ill-conditioning as the runaway steepens.

As a further illustration we also include in Fig. 2 the results of a second case where the reaction enthalpy $H$ is included as an unknown parameter. A minimum of three 
transient measurements is now needed and placing those measurements at the stars shown in Fig. 2 then minimises $\operatorname{cond}\left(\mathbf{V}_{5}\right)$. The final temperature measurement is important in estimating $H$, since by then reaction has completely stopped, while the other two are more favourably placed earlier in the transient in $T(t)$.

\section{Optimising choice of field sensors and their location}

For effective process operation (encompassing monitoring, manual and automatic control) a design choice on the location and number of process measurement sensors is needed, i.e. optimising between operational effectiveness and equipment capital cost.

Consider ideal binary distillation from a still pot and a 6 tray column, modelled on a pilot plant unit in Nottingham's L3 laboratory. Temperatures can be measured on any tray and/or the still pot. Take as a measure of operating effectiveness the accuracy of tray and still pot composition estimates from a Kalman filter fed with the measured temperatures (in fact we included relative volatility as an unknown parameter for estimation along with the plant state). What performance trade-off is there between operating/estimator performance and the number and location of the temperature sensors installed?

A suitable model under the usual simplifying assumptions involves tray and still pot compositions and still pot hold-up plus the unknown 'relative volatility' parameter (9 state variables). The measurement vector includes a direct still pot level measurement plus any of 167 temperature sensor combinations (i.e. 7 alternative single sensors through all combinations to the full set of 7 sensors). With the system at total reflux the state space model is linear invariant allowing calculation of $\mathbf{V}$ via Equation 3.

The sensor locations which minimise $\operatorname{cond}(\mathbf{V})$ for the size of each sensor set (from 1 to all 7) is given in Table 1. In each case, the asymptotic composition estimation error achieved using a Kalman filter fed with measurements from the optimum sensor combination is also quoted (in terms of the trace of the square root of the estimation error covariance matrix $\mathbf{G})$. With more sensors, $\operatorname{cond}(\mathbf{V})$ falls gradually with the

Table 1. Optimum tray locations for different numbers of temperature sensors in a 6 tray batch distillation unit (the stillpot is Tray 0) and unknown relative volatility.

\begin{tabular}{cccc}
\hline No of sensors & $\begin{array}{c}\text { Optimum location of } \\
\text { sensors (Tray Nos) }\end{array}$ & $\begin{array}{c}\text { Minimum } \\
\log (\operatorname{cond}(\mathbf{V}))\end{array}$ & $\operatorname{Trace}(\sqrt{ } \mathbf{G})$ \\
\hline 1 & $0^{\mathrm{a}}$ & $6.62^{\mathrm{a}}$ & 1.436 \\
2 & 04 & 5.21 & 0.374 \\
3 & 024 & 3.71 & 0.283 \\
4 & 0236 & 2.96 & 0.281 \\
5 & 01236 & 2.73 & 0.272 \\
6 & 012345 & 2.48 & 0.265 \\
7 & 0123456 & 2.48 & 0.262 \\
\hline
\end{tabular}

a The Relative volatility is unobservable with only one sensor and the $\operatorname{cond}(\mathbf{V})$ value quoted is that for the tray and still pot compositions only (which are observable). 
composition estimation error, the relative improvements diminishing progressively. Moving from 2 to 3 sensors, which more easily sense curvature in the equilibrium line, significantly improves accuracy of the unknown relative volatility estimates.

State estimation in batch distillation been addressed in more detail by a number of workers, including Oisiovici and Cruz (2000) who recently considered a multicomponent case. They observed that alternative sensor locations lead to varying estimation accuracy, but they provided no guidance on how a choice of the number or location of sensors can be made in general. Here, however, we have demonstrated that $\operatorname{cond}(\mathbf{V})$ can be used to provide that general guidance.

\section{Conclusions}

Based on the results presented we conclude that $\operatorname{cond}(\mathbf{V})$ provides a non-subjective metric for optimising measurement strategies in several areas of process engineering.

In parameter estimation, and especially in the case of chemical reaction kinetics determination, we have demonstrated how this metric can be used to define the timing for capture of a minimal set of measurements in an experimental run. In minimising the number of measurement points needed we minimise overheads in terms of both manpower costs and resource usage in laboratory analysis and data processing. This is of particular significance in a front-end process development context.

When applied to specifying temperature sensors for use in batch distillation we have shown that $\operatorname{cond}(\mathbf{V})$ provides a criterion for choice of both the number and location of sensors within a batch column system to balance against the benefit from higher quality process information and the results of this have been confirmed by comparison with the performance of a state estimation. This is of significance in both distillation processes and the wider context of sensor system design.

Finally, in the case studies reported we have also validated alternative strategies for calculation of the observability matrix itself, including one for non-linear applications.

\section{References}

Mossebach, M., and Reichert, K-H., 1997, Adiabatic reaction calorimetry for data acquisition of free radical polymerisations. J App Poly Sci, 66, 673-681.

Muske, K.R. and Georgakis, C., 2003, Optimal measurement system design for chemical processes, AIChE Journal, 49, 6, 1488-1494.

Oisiovici, R.M. and Cruz, S.L., 2000, State estimation of batch distillation columns using an extended Kalman filter, Chem Eng Sci, 55, 4667-4680.

Ray, W.H., 1981, Advanced Process Control. McGraw-Hill, New York.

Waldraff, W., Dochain, D., Bourrel, S. and Magnus, A., 1998, On the use of observability measures for sensor location in tubular reactor, J Proc Cont, 8, 5\&6, 497-505.

\section{Acknowledgements}

S Y Guhe is grateful for support from the University of Nottingham in contributing to the work reported. 